\title{
Engineering of Porous Silica Coated Gold Nanorods by Surface-protected Etching and Their Applications in Drug Loading and Combined Cancer Therapy
}

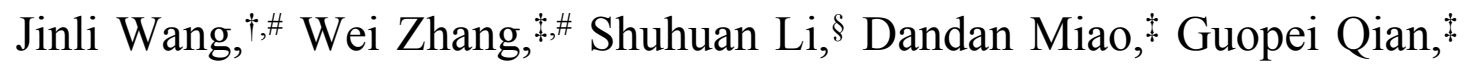

Gaoxing $\mathrm{Su}^{*}$,

'Department of Pharmacy, Affiliated Hospital of Nantong University, Nantong 226001, China

\$School of Pharmacy, Nantong University, Nantong 226001, China

§Department of Food Science and Engineering, Shandong Agriculture and Engineering University, Jinan 251100, China

Corresponding Author: sugaoxing@ntu.edu.cn (G. Su)

\#These two authors contributed equally to this work.

The Supporting Information file includes 6 figures.

Figure S1. $\mathrm{N}_{2}$ adsorption-desorption isotherm curves of GNR@p-SiO ${ }_{2}$ (Page S2)

Figure S2. Hydrodynamic diameters and Zeta potentials of nanoparticles. (Page S2)

Figure S3. Stability of nanoparticles. (Page S3)

Figure S4. Fluorescence spectra of drug-loaded nanoparticles. (Page S3)

Figure S5. Cell viabilities of the HeLa cells after different treatments. (Page S4)

Figure S6. The pharmacokinetics and biodistribution of nanoparticles. (Page S4) 




Figure S1. $\mathrm{N}_{2}$ adsorption-desorption isotherm curves of GNR@p-SiO $\mathrm{S}_{2}$ and their corresponding pore size distributions (inset).
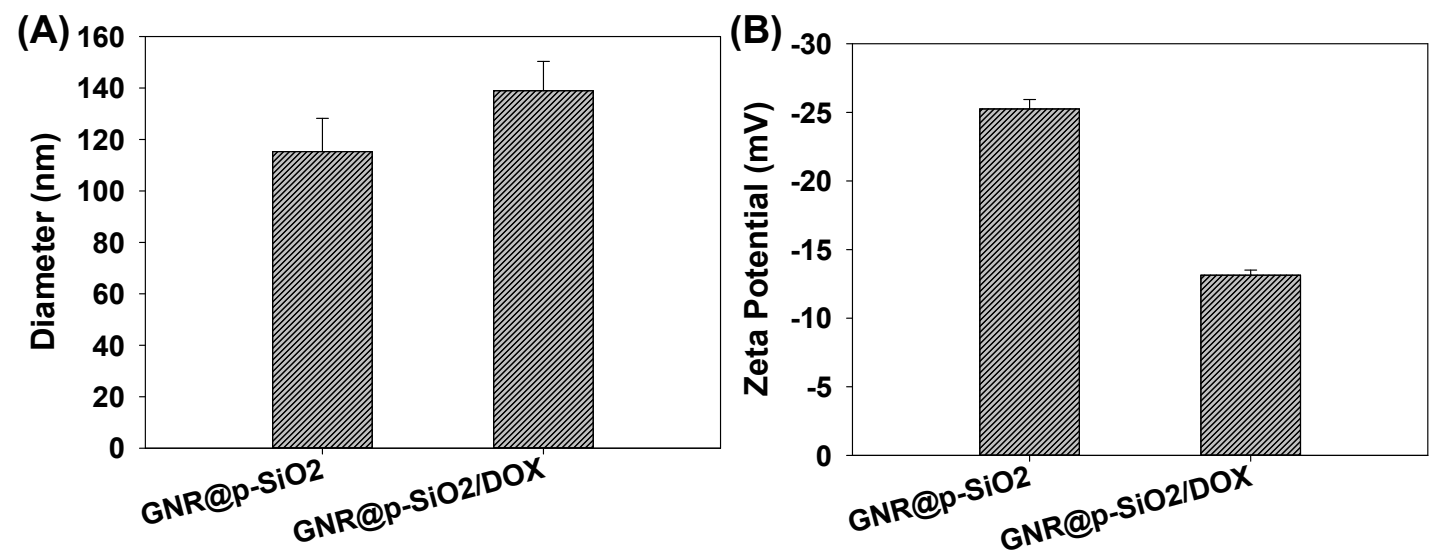

Figure S2. (A) Hydrodynamic diameters of GNR@p-SiO $\mathrm{S}_{2}$ and GNR@p-SiO $2 / \mathrm{DOX}$. (B) Zeta potentials of GNR@p-SiO 2 and GNR@p-SiO $2 / \mathrm{DOX}$. 

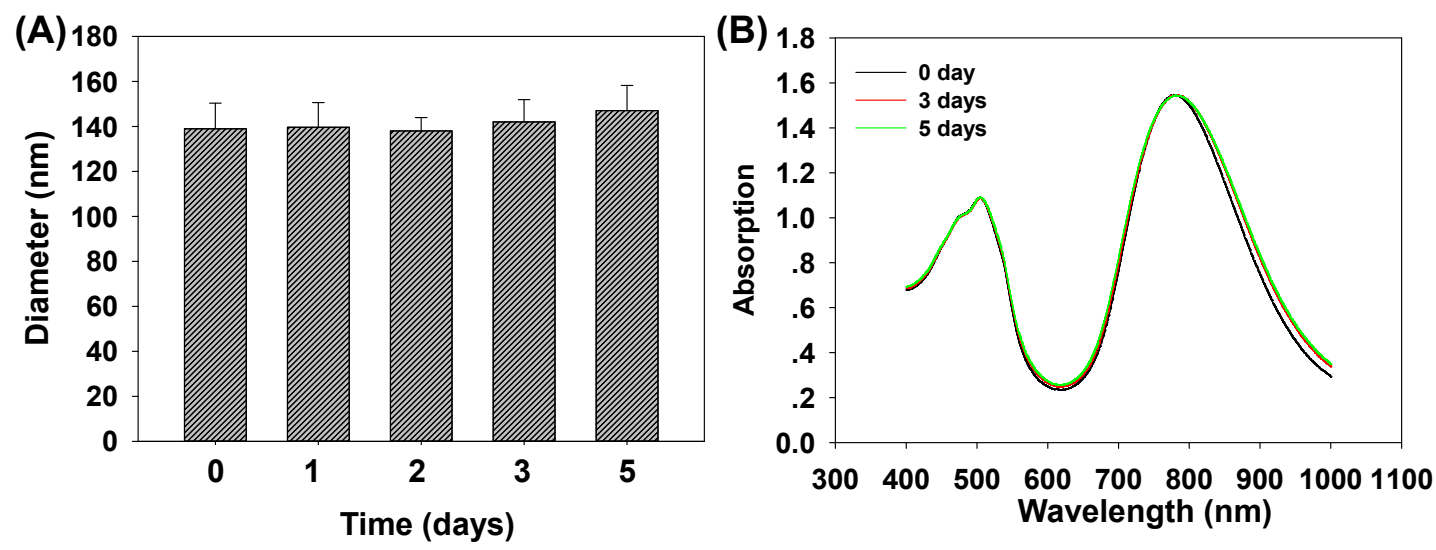

Figure S3. Stability of GNR@p- $\mathrm{SiO}_{2} / \mathrm{DOX}$. (A) Hydrodynamic diameters and (B) UV-Vis-NIR absorption spectra of GNR@p- $\mathrm{SiO}_{2} / \mathrm{DOX}$ after dispersing into PBS for different days.

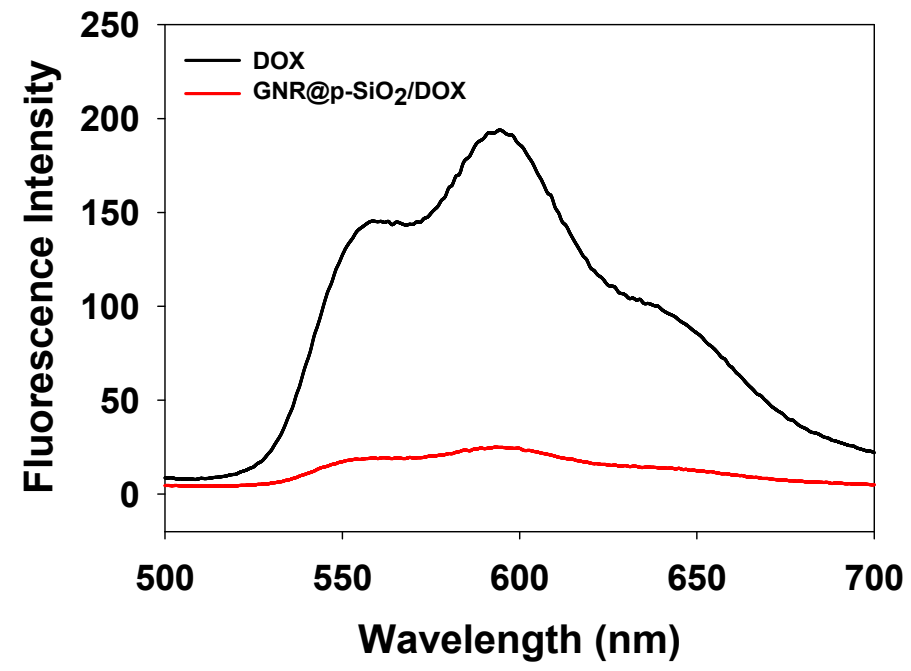

Figure S4. Fluorescence spectra of DOX and GNR@p-SiO ${ }_{2} / \mathrm{DOX}$. 

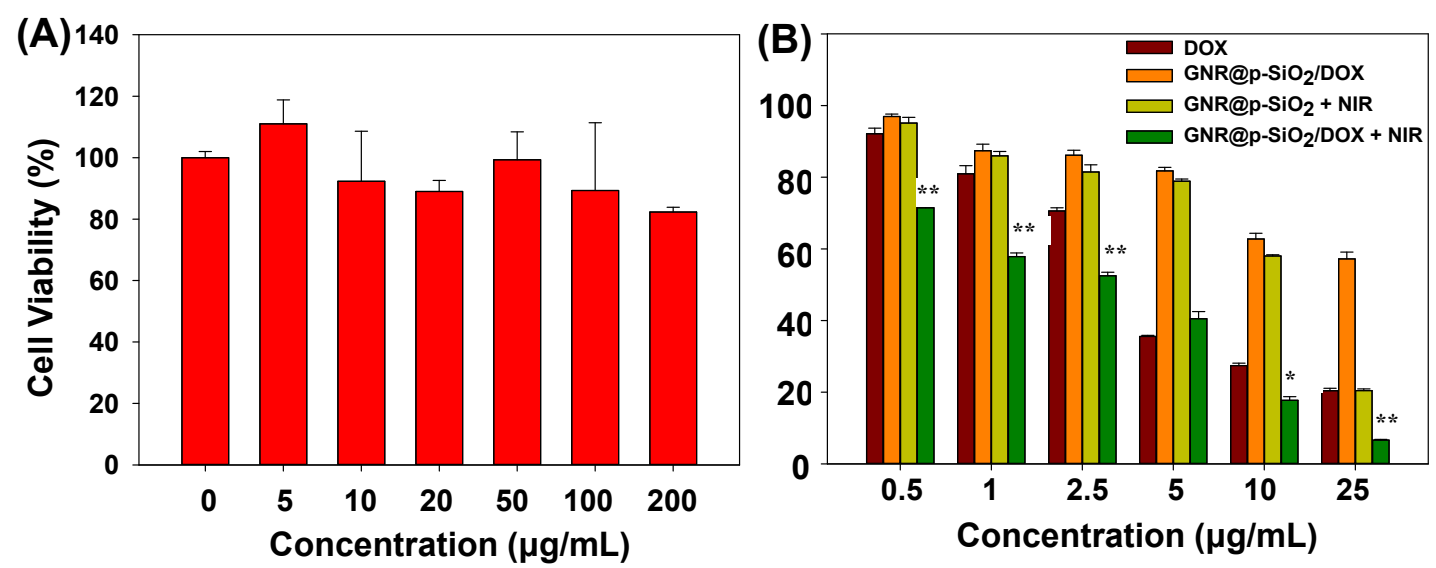

Figure S5. (A) Cell viabilities of the HeLa cells after incubation with various concentrations of GNR@p-SiO 2 . (B) Cell viabilities of HeLa cells after incubation with different concentrations of free DOX, GNR@p-SiO 2 and GNR@p-SiO $2 / \mathrm{DOX}$ with or without NIR irradiation. All groups had equivalent concentrations of DOX or nanoparticles. Data were presented as means $\pm \mathrm{SD}(\mathrm{n}=3)$. $* \mathrm{P}<0.05$, $* * \mathrm{P}<0.01$.

(A)

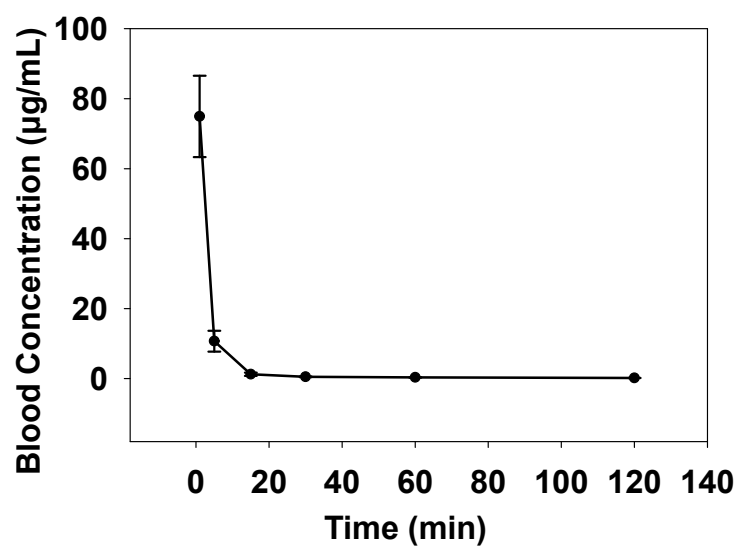

(B)

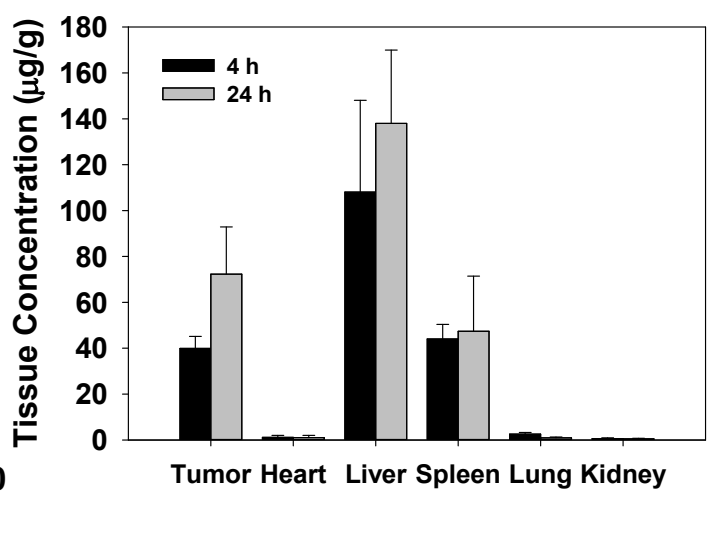

Figure S6. (A) The blood concentration-time curve in mice plasma after i.v. administration of $\mathrm{GNR} @ \mathrm{p}-\mathrm{SiO}_{2}$. (B) Biodistribution of $\mathrm{GNR} @ \mathrm{p}-\mathrm{SiO}_{2}$ in tumor-bearing mice. 